Research Article

\title{
Prevention of Mother-to-Child HIV Transmission Service Utilization among Pregnant Women in Northeast Ethiopia: A Cross-Sectional Survey
}

\author{
Betregiorgis Zegeye $\mathbb{D}^{1},{ }^{1}$ Gorems Lemma, ${ }^{1}$ Abebe Balcha, ${ }^{1}$ \\ and Mitku Mammo Taderegew $\mathbb{1}^{2}$ \\ ${ }^{1}$ Department of Public Health, College of Health Science, Debre Berhan University, Debre Berhan, Ethiopia \\ ${ }^{2}$ Department of Biomedical Sciences, College of Medicine and Health Sciences, Wolkite University, Wolkite, Ethiopia \\ Correspondence should be addressed to Mitku Mammo Taderegew; mitkumamo@gmail.com
}

Received 12 May 2020; Revised 2 October 2020; Accepted 8 October 2020; Published 4 November 2020

Academic Editor: Mohammed N. Al Ahdal

Copyright (c) 2020 Betregiorgis Zegeye et al. This is an open access article distributed under the Creative Commons Attribution License, which permits unrestricted use, distribution, and reproduction in any medium, provided the original work is properly cited.

\begin{abstract}
Background. Targeting pregnant women attending antenatal care clinics provides a unique opportunity for implementing the Prevention of Mother-to-Child Transmission (PMTCT) programs against human immunodeficiency virus (HIV) infection of newborn babies. The objective of this study was to assess the PMTCT service utilization rate and to characterize its reasons among pregnant women attending antenatal care clinics at selected public health facilities in Debre Berhan Town, Northern Ethiopia. Methods. A facility-based cross-sectional survey was conducted among 355 pregnant women from May 1 to June 15, 2019. The participants were selected by systematic random sampling technique, and data were collected using a pretested intervieweradministered structured questionnaire. Descriptive statistics like frequency, mean, and standard deviation were reported using text, table, and graphs. Results. The mean ages of the respondents were $24( \pm 5.6)$ years, and the majority of the respondents $(287$ $(80.8 \%)$ ) were urban residents. In this study, prevention of mother-to-child HIV transmission service utilization rate was $86.8 \%$. The most frequently mentioned reasons for not utilization of services were fear of stigma and discrimination (42.6\%), fear of rejection by partner (19.1\%), fear of positive test results (17.0\%), lack of awareness (12.7\%), and wastage of time (8.5\%). Hence, continuous health education and comprehensive counseling are necessary to increase the awareness and reduce stigma, fear of the positive result, and partner rejection.
\end{abstract}

\section{Introduction}

Mother-to-child transmission (MTCT) of human immunodeficiency virus (HIV) defined as the transmission of HIV from an HIV-positive mother to her baby at the time of pregnancy, labor, delivery, or breastfeeding $[1,2]$. It is the most communal way that children become infected with HIV $[1,3]$. Ninety-five percent of HIV-infected children acquired the infection through mother-to-child transmission during pregnancy, around the time of labor and delivery or during breastfeeding [4].

MTCT responsible for the vast majority of more than 700,000 estimated new HIV infections in children worldwide annually [5]. In 2015, there were approximately 2.1 million new HIV infections, 150,000 of which were among children. Most of these children were from subSaharan Africa, and the disease was acquired via their HIVinfected mothers at the time of pregnancy, delivery, or breastfeeding [6]. Despite the improvement made in the decline of MTCT in Ethiopia by enhancing the availability of HIV counseling and testing (HCT) services for pregnant women, low percentages of pregnant women receive the PMTC Services. A total of 400,000 mothers with reproductive age and 62,000 children aged 0 to 14 years with HIV-infection were reported in the country in the year 2016. Still, the percentage of pregnant women counseled 
and tested for PMTCT during an ANC visit or labor was $34 \%[7,8]$.

The PMTCT plays a determinant role in minimizing the number of children being infected with HIV. Without any interference, $20-50 \%$ of infants would be infected with HIV; $5-10 \%$ during pregnancy, $10-20 \%$ during labor and delivery, and $5-20 \%$ through breastfeeding. By realizing the PMTCT program, the overall risk can be minimized to less than $2 \%$ [9].

Several influences lead to the low utilization of PMTCT services. Decreasing in the utilization of ANC, skilled birth attendants, and HIV counseling and testing (HCT) can affect the PMTCT service utilization [10]. Even if the number of health facilities offering PMTCT service has increased dramatically in Ethiopia, MTCT of HIV still persisting to be a challenge for the country may be due to high missed opportunities, dropout rates, and low utilization of services [11]. According to Ethiopia Demographic and Health Survey (EDHS) 2011 report, there is still a huge gap between the national ANC coverage (34\%) and the percentage of women, who were counseled and tested and received the result during ANC (11\%) [12]. Later on, the EDHS 2016 report showed that, nationally, the percentage of women who were counseled tested and received the result during ANC was $21.9 \%$ [8]. Particularly, in the Amhara region, the coverage indicates $31.5 \%$ [13].

To the best of our knowledge, there is little information on PMTCT service utilization and its associated factors in the study area. In spite of intensive efforts to scale-up PMTCT services in Ethiopia, the coverage and uptake of the service by the pregnant women remain low. Therefore, this study aimed to assess PMTCT service utilization rate and characterize its reasons among pregnant women following ANC at public health facilities of Debre Berhan Town, Northeast Ethiopia. The result of this study could provide up-to-date information about current level of utilization and its related reasons for low PMTCT services utilization. Such information renders evidence for applying PMTCT services and fills the gaps towards improving HIV prevention and control services, especially in the study area.

\section{Methods and Materials}

2.1. Study Area, Period, and Design. A facility-based crosssectional survey was conducted in Debre Berhan Town, Northeast Ethiopia. Debre Berhan Town is located at about $130 \mathrm{~km}$ away from Addis Ababa, the capital city of Ethiopia. According to the 2015/16 annual statistical report of the town's Finance and Economic Development Office, the total population of the town was 57,787 . There is one public referral hospital and one private primary hospital, 3 health centers, and 9 health posts. The study was conducted from May 1 to June 15, 2019.

2.2. Study Population and Sampling Techniques. The source populations were all pregnant women who attended ANC in the selected public health facilities during the study period and those pregnant women included in the study were considered as the study population. All pregnant women who attended the ANC clinic during the study periods were eligible for the study and those pregnant women who were severely ill and mentally and physically not capable of being interviewed were excluded from the study.

The sample size for this study was calculated using a single population proportion formula by considering a confidence level of $95 \%$, marginal error $5 \%, 31 \%$ proportion of PMTCT utilization from a previous study [13], and 10\% nonresponse rate. Hence, the final calculated sample size was 360.

To select the study participants, first, we took all public health facilities ( 3 health centers and one referral hospital). The total sample was equally divided into those health facilities based on the assumption that ANC and PMTCT service utilization in all those health facilities were not significantly different from each other. Then, the average numbers of ANC visits per day in those health facilities were calculated as 10 as we have visited the ANC register of health facilities. Accordingly, about 200 pregnant women per health facility were expected to attend ANC during the data collection period (20 days). Then, a sampling interval of 2 was obtained by dividing the total expected number of pregnant women to the actual sample size. Thus, every second pregnant woman attending ANC clinics, irrespective of the numbers of previous ANC visits, was selected until the required sample size was achieved (Figure 1).

2.3. Data Collection Procedures. The data were collected using a pretested interviewer-administered structured questionnaire. The questionnaire was prepared by reviewing different published articles $[1,10,11,14,15]$ with similar title in which the cultural and socioeconomic characteristics of study participants were similar to the target population of this study. The tool was primarily prepared in English language and translated to local language (Amharic) by an expert who had a good ability of the two languages and then another person with good ability of both languages retranslated back to English to ensure consistency. The internal validity of the questioner was also assessed by comparing questionnaire responses with objectives of the study during the pretest. The data were collected by trained midwives after taking two days of intensive training about the objectives of the study, tools, and ethical concerns.

The quality of the data was maintained by conducting a pretest on $5 \%$ of the sampled population in Keyit health Center, which was not part of the actual data collection area. Two-day training was given for data collectors and supervisors related to the objective of the study, tools, and ethical concerns. Moreover, the collected data were checked daily to safeguard its completeness.

The collected data were cleaned, coded, entered into EPIinfo version-7 software, and exported to statistical package for social science (SPSS) version 20 for analysis. Descriptive statistics including frequencies, means, and standard deviations were computed to summarize the variables. 


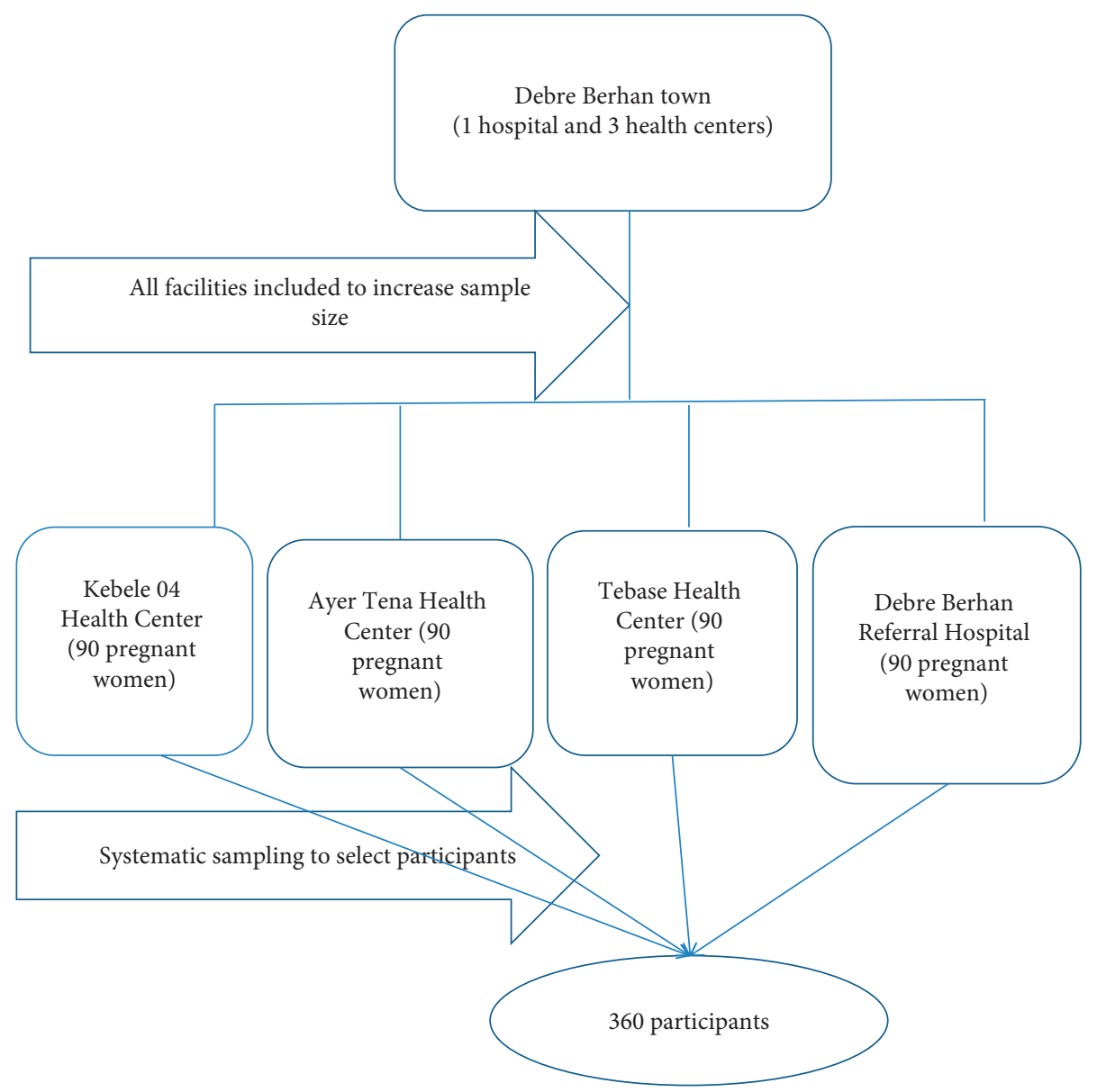

Figure 1: Schematic representation of sampling procedure for assessing PMTCT service utilization in Debre Berhan Town, June, 2019.

2.4. Ethics Approval and Consent to Participate. The study was conducted after ethical letters were obtained from Ethical Review committee of the Department of Public Health, Victory College, Debre Berhan Campus, Ethiopia. Then, permission was taken from hospital and health centers' clinical director offices. The data were collected after obtaining verbal and written informed consent from the study subjects. However, some study participants were under 18 years of age. Thus, consent to participate was collected from the partner, and also assent was obtained from those participants with age less than 18 years. To keep confidentiality, codes were used and unauthorized people did not have access to the data.

\section{Results}

3.1. Sociodemographic Characteristics of the Study Participants. A total of 360 pregnant women who attended the ANC visit were invited, and 355 of them were volunteered to participate in the study making the response rate $98.6 \%$. The mean age of the respondents was $24( \pm 5.6)$ years, and the majority of the respondents $(287(80.8 \%))$ were from urban areas. Out of the total respondents, 298 (83.9\%) were orthodox christian by religion and $42(11.8 \%)$ were muslims. The majority of the respondents $(98.0 \%)$ were currently married. Nearly half of the respondents (174 (49.0\%)) were housewives and 108 (30.4\%) of their husbands were merchants by occupation. Regarding educational status, the majority 259 (72.9\%) of the respondent's educational status was secondary and above, and fifty-eight (16.3\%) of the respondents had no formal education (Table 1).

3.2. Obstetric Characteristics of the Respondents. Among the total of the respondents, $218(61.4 \%)$ were multigravida and only $17(7.8 \%)$ of them had no ANC visits in their last pregnancy. Of those respondents who gave birth previously, $186(85.3 \%)$ were delivered at the health facilities. Nearly half of the respondents $(51.0 \%)$ came with their partner and about $162(45.6 \%)$ of the respondents discussed with their partner about HIV/AIDS. The majority (326 (91.8\%)) respondents arrived at a health facility with less than 30 minutes. From all $308 \mathrm{HIV}$-tested pregnant women, the majority of them (96.1\%) came with their partners for ANC follow-up visits. And, out of all the pregnant women who were not tested, most of them (85.1\%) did not involve their partners (Table 2).

3.3. Knowledge about PMTCT. In this study, among all of the respondents, $330(93.0 \%)$ respondents heard about MTCT of HIV. Three-hundred twenty (85.4\%) participants knew PMTCT of HIV. Of these, 250 (70.4\%) of the respondents 
TABLE 1: Sociodemographic characteristics of pregnant women attending ANC at public health facilities, Debre Berhan Town, June 2019 $(N=355)$.

\begin{tabular}{|c|c|c|c|}
\hline Variable & Category & Frequency $(N=335)$ & Percent \\
\hline \multirow{3}{*}{ Age (years) } & $15-24$ & 201 & 56.6 \\
\hline & $25-34$ & 98 & 27.6 \\
\hline & $\geq 35$ & 56 & 15.8 \\
\hline \multirow{4}{*}{ Ethnicity } & Amhara & 278 & 78.3 \\
\hline & Oromo & 57 & 16.1 \\
\hline & Gurage & 14 & 3.9 \\
\hline & Tigre & 6 & 1.7 \\
\hline \multirow{3}{*}{ Religion } & Orthodox christian & 298 & 83.9 \\
\hline & Muslim & 42 & 11.8 \\
\hline & Protestant and others & 15 & 4.3 \\
\hline \multirow{4}{*}{ Educational status } & No formal education & 58 & 16.3 \\
\hline & Elementary & 38 & 10.7 \\
\hline & Secondary school & 61 & 17.2 \\
\hline & Diploma and above & 198 & 55.7 \\
\hline \multirow{5}{*}{ Occupational status } & Professional & 18 & 5.1 \\
\hline & Semiprofessional & 39 & 11.0 \\
\hline & Skilled & 82 & 23.1 \\
\hline & Semiskilled & 88 & 24.7 \\
\hline & Manual & 128 & 36.1 \\
\hline \multirow{2}{*}{ Current marital status } & Married & 348 & 98.0 \\
\hline & Divorced & 7 & 2.0 \\
\hline \multirow{2}{*}{ Resident } & Urban & 287 & 80.8 \\
\hline & Rural & 68 & 19.2 \\
\hline \multirow{4}{*}{ Partner's occupation } & Farmer & 27 & 7.6 \\
\hline & Government employee & 19 & 5.4 \\
\hline & Merchant & 108 & 30.4 \\
\hline & Other & 201 & 56.6 \\
\hline
\end{tabular}

TABLE 2: Obstetrics and other factors among pregnant women attending ANC at public health facilities, Debre Berhan Town, June 2019 $(N=355)$.

\begin{tabular}{|c|c|c|c|}
\hline Variable & Category & Frequency & Percent \\
\hline \multirow{3}{*}{ Time taken to arrive at the health facility } & $<30 \mathrm{~min}$ & 326 & 91.8 \\
\hline & $30-1 \mathrm{hr}$ & 22 & 6.2 \\
\hline & $>1 \mathrm{hr}$ & 7 & 2.0 \\
\hline \multirow{4}{*}{ Number of pregnancies } & 1 & 137 & 38.6 \\
\hline & 2 & 56 & 15.8 \\
\hline & 3 & 71 & 20.0 \\
\hline & $\geq 4$ & 91 & 25.6 \\
\hline \multirow{2}{*}{ ANC in last pregnancy } & No & 17 & 7.8 \\
\hline & Yes & 201 & 92.2 \\
\hline \multirow{3}{*}{ Place of last delivery } & Home & 10 & 4.9 \\
\hline & HF & 186 & 88.5 \\
\hline & Others & 14 & 6.6 \\
\hline \multirow{4}{*}{ Number of ANC visits in the current pregnancy } & One & 72 & 20.3 \\
\hline & Two & 78 & 22.0 \\
\hline & Three & 149 & 42.0 \\
\hline & Four and above & 56 & 15.8 \\
\hline \multirow{2}{*}{ Partner involvement in ANC } & No & 174 & 49.0 \\
\hline & Yes & 181 & 51.0 \\
\hline \multirow{2}{*}{ Discussion with a partner about HIV } & No & 162 & 45.6 \\
\hline & Yes & 193 & 54.4 \\
\hline
\end{tabular}

Note. ANC: antenatal care; HIV: human immunodeficiency virus. 
TABLE 3: Knowledge-related factors among pregnant women attending ANC at public health facilities, Debre Berhan Town, June 2019 $(N=355)$.

\begin{tabular}{|c|c|c|c|}
\hline Variable & Category & Frequency & Percent \\
\hline \multirow{2}{*}{ Heard about MTCT of HIV } & No & 25 & 7.0 \\
\hline & Yes & 330 & 93.0 \\
\hline \multirow{3}{*}{ Source of information } & Social/mass media & 90 & 25.4 \\
\hline & Health professional & 242 & 68.2 \\
\hline & Others & 23 & 6.4 \\
\hline \multirow{3}{*}{ HIV can be transmitted during pregnancy } & No & 42 & 11.8 \\
\hline & Yes & 309 & 87.0 \\
\hline & I do not know & 4 & 1.1 \\
\hline \multirow{3}{*}{ HIV can transmitted during labor } & No & 40 & 11.3 \\
\hline & Yes & 298 & 83.9 \\
\hline & Do not know & 16 & 4.5 \\
\hline \multirow{3}{*}{ HIV can transmitted during lactation } & No & 45 & 12.6 \\
\hline & Yes & 293 & 82.5 \\
\hline & Do not know & 17 & 4.9 \\
\hline \multirow{3}{*}{ ANC important for PMTCT } & No & 22 & 6.2 \\
\hline & Yes & 317 & 89.3 \\
\hline & Do not know & 16 & 4.5 \\
\hline \multirow{3}{*}{ Health facility delivery important for PMTCT } & No & 24 & 6.8 \\
\hline & Yes & 314 & 88.5 \\
\hline & Do not know & 17 & 4.8 \\
\hline \multirow{3}{*}{ Breastfeeding counseling important for PMTCT } & No & 35 & 9.9 \\
\hline & Yes & 312 & 87.9 \\
\hline & Do not know & 8 & 2.3 \\
\hline \multirow{2}{*}{ HIV test done at $\mathrm{HC} /$ hospital } & No & 21 & 5.9 \\
\hline & Yes & 334 & 94.1 \\
\hline \multirow{3}{*}{ ART can reduce MTCT of HIV } & No & 48 & 13.5 \\
\hline & Yes & 250 & 70.4 \\
\hline & I do not know & 57 & 16.1 \\
\hline
\end{tabular}

knew ART drugs given for HIV-positive pregnant mothers could reduce the risk of HIV transmission (Table 3).

3.4. Utilization of PMTCT Services. Out of 355 respondents, 346 (97.5\%) were precounseled and 308 (86.8\%) were tested for HIV. Among respondents who were tested for HIV, 147 $(47.7 \%)$ have been tested in the current visit, and the rest (161 (52.3\%)) have been tested three months ago. Among those tested respondents, majority of them (303 (98.4\%)) and two-hundred eighty-nine (95.4\%) received their result and negative test results, respectively (Table 4 ).

3.5. Reasons for Not Utilizing PMTCT Services. Among 47 pregnant women who refused for HIV testing, nearly half of them $(42.6 \%)$ were due to fear of stigma and discrimination followed by fear of rejection by partner (19.1\%) (Figure 2).

\section{Discussion}

This study assessed the utilization of PMTCT services and the reasons for not using PMTCT services in selected public health facilities of Debre Berhan Town, Northeast Ethiopia. As part of ANC service, the study showed potential areas for the improvement of PMTCT service improvements. The primary sources of information about ANC for respondents in this study were health professionals including health extension workers. This could be partly because the majority of the respondents were from urban areas and had access to health facilities with less than 30 minutes of travel.

In the present study, the PMTCT service utilization among ANC attendants was found to be $86.8 \%$. This finding was lower as compared to that of a previous study conducted in Addis Ababa, central Ethiopia, which showed 94\% of pregnant women accepted HIV counseling and testing [10]. This could be because more respondents of our study who visited the health facilities came from rural areas as compared to the respondents who were in Addis Ababa. Utilization of PMTCT service in this study is similar to a study conducted in Sebeta, Central Ethiopia [11] which indicated that $86.9 \%$ of the respondents reported that they had been tested for HIV during the study periods either in the selected health facility or elsewhere. Moreover, our finding was nearly similar to a study conducted in Oromia regional state, Ethiopia, in 2016 which showed a service utilization rate of $83 \%$ [1]. However, the result of our study is higher than that of the EDHS 2016 report in Amhara regional state of Ethiopia, which indicated that only $31.5 \%$ of the respondents were counseled and tested and received results. This might be because most respondents in this study were urban residents, and there might be some service improvement as well as an increase in the level of awareness through time [8]. 
TABLE 4: HIV testing and counseling as PMTCT service utilization among pregnant women attending ANC at public health facilities, Debre Berhan Town, June $2018(n=355)$.

\begin{tabular}{|c|c|c|c|}
\hline Variable & Category & Frequency & Percent \\
\hline \multirow{2}{*}{ Have got precounseling } & No & 9 & 2.5 \\
\hline & Yes & 346 & 97.5 \\
\hline \multirow{2}{*}{ Tested HIV } & No & 47 & 13.2 \\
\hline & Yes & 308 & 86.8 \\
\hline \multirow{2}{*}{ Received test result } & No & 5 & 1.6 \\
\hline & Yes & 303 & 98.4 \\
\hline \multirow{3}{*}{ Result of the test } & Positive & 3 & 1.0 \\
\hline & Negative & 289 & 95.4 \\
\hline & Do not know & 11 & 3.6 \\
\hline \multirow{2}{*}{ Have got posttest counseling } & No & 5 & 1.6 \\
\hline & Yes & 303 & 98.4 \\
\hline \multirow{2}{*}{ Linked to ART } & No & 0 & 0 \\
\hline & Yes & 3 & 100 \\
\hline \multirow{2}{*}{ Readiness to go ART clinic right now } & No & 0 & 0 \\
\hline & Yes & 3 & 100 \\
\hline \multirow{2}{*}{ Satisfaction by service } & No & 86 & 24.2 \\
\hline & Yes & 269 & 75.5 \\
\hline
\end{tabular}

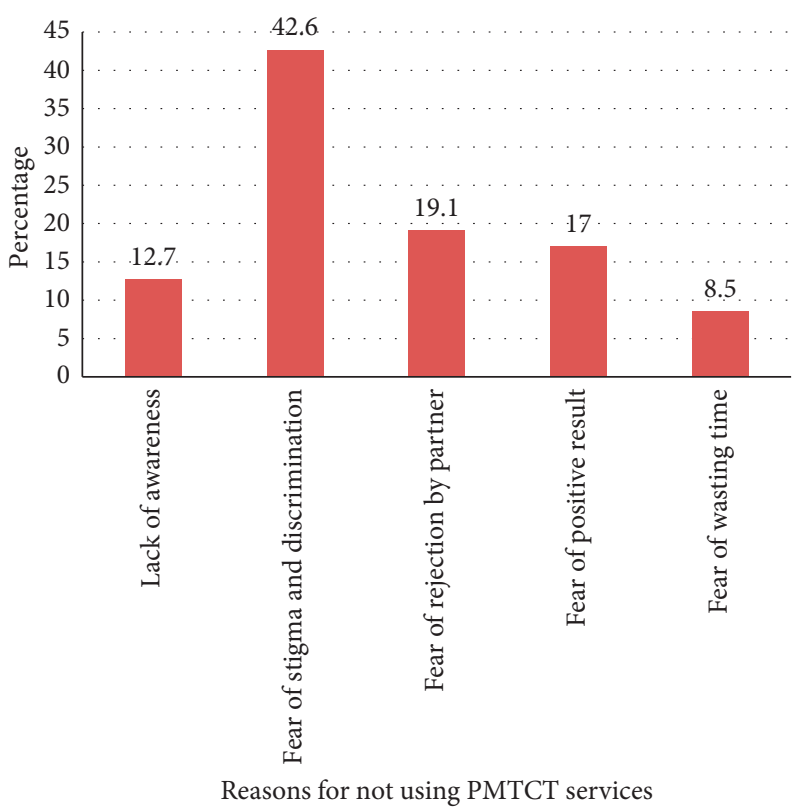

Figure 2: Reasons for not using PMTCT services among pregnant women attending ANC at public health facilities, Debre Berhan Town, June 2019.

This study also assessed the reasons for not utilizing the PMTCT services. Fear of stigma and discrimination, fear of rejection by a partner, fear of positive result, lack of awareness, and wastage of time were the most common reasons reported by the study participants for refusals of PMTCT service utilization. In line with our findings, a study conducted in Sebeta showed that waiting for a long time for the service and lack of awareness and knowledge about the MTCT and PMTCT service utilization were the commonly mentioned reasons [1].
This study also agreed with the study in Addis Ababa, indicating that the lack of awareness about the availability and benefits of ANC/PMTCT services, shortage of PMTCT service providers, lack of adequate and separate room for PMTCT services, poor involvement of partners/husbands in ANC/PMTCT services, poor disclosure of HIV-status to partners, and psychological unpreparedness due to fear of being positive for HIV were the main barriers preventing the mothers from HIV testing [7]. 
Due to descriptive nature of study design used, it was not possible to correlate variables and to determine the cause and effect relationships which was the limitation of the study.

\section{Conclusions}

In this study, the PMTCT service utilization among ANC attendees was found to be $86.8 \%$. This indicates that utilization of PMTCT services was low compared to the national direction of providing the services to all ANC attendants. The most frequently mentioned reasons for no utilization of PMTCT services were fear of stigma and discrimination followed by fear of rejection by the partners. It is very important to strengthen the health information provision about PMTCT services and broaden the channels of communication to reach a wider audience for conveying the needed information to decrease stigma and discrimination in the community. In addition, the involvement of partners is needed, and providing comprehensive couple counseling is also required to avoid result-related fear and to reduce partner rejection fears.

\section{Data Availability}

The datasets used and/or analyzed during this study are available from the corresponding author on reasonable request.

\section{Conflicts of Interest}

The authors declare that they have no conflicts of interest.

\section{Acknowledgments}

The authors would like to extend their deepest appreciation to the staff members of Debre Berhan Referral Hospital, Kebele 04 Health Center, Tebase Health Center, and Ayer Tena Health Center for their cooperation and providing the necessary information for this study. We also would like to thank all study participants for their cooperation.

\section{References}

[1] A. Feyera, B. E. Megerssa, D. Legesse, and F. A. Hailemichael, "Prevention of mother to child transmission of HIV/AIDS: service utilization and associated factors among selected public health facilities in Ethiopia," Medical Practice and Reviews, vol. 8, no. 11, pp. 1-3, 2017.

[2] M. A. Tolle and D. Dewey, "Prevention of mother-to-child transmission of HIV infection," HIV Curriculum for the Health Professional, Baylor International Pediatric AIDS Initiative, Houston, TX, USA, 2010.

[3] World Health Organization, Global HIV/AIDS Response: Epidemic Update and Health Sector Progress towards Universal Access: Progress Report, World Health Organization, Geneva, Switzerland, 2011, https://apps.who.int/iris/handle/10665/ 44787.

[4] A. Global, Information and Education on HIV and AIDS. Prevention of Mother-to-Child Transmission (PMTCT) of HIV,
2018, https://www.avert.org/professionals/hiv-programming/ prevention/prevention-mother-child.

[5] Federal Democratic Republic of Ethiopia, "Country Progress Report on the HIV Response," 2014, https://www.unaids.org/ sites/default/files/country/documents/ETH_narrative_ report_2014.pdf.

[6] World Health Organization, PMTCT Strategic Vision 20102015: Preventing Mother-To-Child Transmission of HIV to Reach the UNGASS and Millennium Development Goals: Moving towards the Elimination of Paediatric HIV, World Health Organization, Geneva, Switzerland, 2009, https://apps. who.int/iris/bitstream/handle/10665/44268/9789241599030_ eng.pdf.

[7] World Health Organization, "HIV Country profile Ethiopia 2016," World Health Organization, Geneva, Switzerland, 2016, https://www.who.int/hiv/data/Country_profile_ Ethiopia.pdf?ua=1.

[8] Central Statistical Agency (CSA) (Ethiopia) and ICF, Ethiopia Demographic and Health Survey 2016, CSA and ICF, Addis Ababa, Ethiopia, and Rockville, MD, USA, 2016, https:// dhsprogram.com/pubs/pdf/SR241/SR241.pdf.

[9] World Health Organization, Guidance on Global Scale-Up of the Prevention of Mother-Child Transmission of HIV: Towards Universal Access for Women, Infants and Young Children and Eliminating HIV and AIDS among Children, World Health Organization, Geneva, Switzerland, 2007, http://apps.who.int/ iris/bitstream/10665/43728/1/9789241596015_eng.pdf.

[10] W. Deressa, A. Seme, A. Asefa, G. Teshome, and F. Enqusellassie, "Utilization of PMTCT services and associated factors among pregnant women attending antenatal clinics in Addis Ababa, Ethiopia," BMC Pregnancy and Childbirth, vol. 14, no. 1, pp. 1-13, 2014.

[11] H. Merga, K. Woldemichael, and L. Dube, "Utilization of prevention of mother-to-child transmission of HIV services and associated factors among antenatal care attending Mothers in Sebeta town, Central Ethiopia," Advances in Public Health, vol. 2016, Article ID 6250898, 10 pages, 2016.

[12] S. M. Tarekegn, L. S. Lieberman, and V. Giedraitis, "Determinants of maternal health service utilization in Ethiopia: analysis of the 2011 Ethiopian Demographic and Health Survey," BMC Pregnancy and Childbirth, vol. 14, no. 1, 161 pages, 2014.

[13] Federal Democratic Republic of Ethiopia Ministry of Health. Health and Health Related Indicators, 2016, http://repository. iifphc.org/bitstream/handle/123456789/395/Health\%20and\% 20Health\%20Related\%20Indicator\%202017.pdf? sequence $=1$ \&isAllowed $=y$.

[14] A. Z. Bissek, I. E. Yakana, F. Monebenimp et al., "Knowledge of pregnant women on mother-to-child transmission of HIV in Yaoundé," The Open AIDS Journal, vol. 5, no. 1, pp. 25-8, 2011.

[15] A. Demissie, A. Deribew, and M. Abera, "Determinants of acceptance of voluntary HIV testing among antenatal clinic attendees at Dil Chora Hospital, Dire Dawa, East Ethiopia," Ethiopian Journal of Health Development, vol. 23, no. 2, 2009. 